\title{
Estudio del plomo medieval en las vidrieras del monasterio de Pedralbes (Barcelona)
}

\author{
Study of the medieval lead cames used in the stained glass \\ windows of the Monastery of Pedralbes (Barcelona)
}

\author{
Fecha de recepción: $28-$ VII-99 \\ Fecha de accptación: 14-X-99

\section{RESUMEN} \\ Se lleva a cabo un estudio de las vidrieras del Monasterio \\ de Pedralbes, en Barcelona, en lo concerniente al plomo, \\ como elemento principal de las mismas.
}

\author{
F.CORTÉS PIZANO \\ Glasmalerei Peters, Paderborn
}

AI.EMANIA

\section{SUMMARY}

This work includes an study of the glass windows of the "Monasterio de Pedralbes", in Barcelona, concerning lead as their main element.

\section{INTRODUCCIÓN}

Las razones por las que nos decidimos a realizar un estudio sobre el plomo medieval de las vidrieras del Monasterio de Pedralbes (Barcelona) son varias. En primer lugar, por la importancia de estas vidrieras medievales para el estudio de esta especialidad en España y especialmente en Cataluña. En segundo lugar, el hecho de que hayan conservado una red de plomo medieval, constituye un hallazgo excepcional en España. En tercer lugar por la absoluta carencia que existe en nuestro país de estudios sobre aspectos técnicos de las vidrieras. Este estudio está basado en métodos de trabajo detectado en las vidrieras, su estado de conservación, aspectos artísticos y estéticos, el proceso de restauración efectuado y la analítica realizada, y tiene como objetivo el mejorar nuestro conocimiento sobre el proceso de trabajo de los vidrieros durante la Edad media y sobre los escasos plomos medievales de vidrieras conservados en España.

\section{EL MONASTERIO DE SANTA MARÍA DE PEDRALBES (BARCELONA)}

El Monasterio de Santa María de Pedralbes, situado a las afueras de Barcelona, fue construido hacia 1328 por iniciativa Real y consagrado a la Orden de las Clarisas. Dado su carácter monástico y de clausura, el monasterio ha conservado, entre otras muchas obras de arte de varios períodos, un conjunto excepcional de vidrieras, la mayoría del siglo XIV (hacia 1330-1340) (1).

Carecemos hasta la fecha de datos procedentes de los archivos del monasterio, los cuales posiblemente nos ofrecerían información más detallada sobre su autoría, datación, intervenciones posteriores, etc. No obstante, lo que es prácticamente seguro es que las vidrieras del XIV del monasterio de Pedralbes, dada su homogeneidad de estilo, su iconografia y técnica, fueron realizadas en un corto período de tiempo y pueden ser atribuidas a un 
mismo autor. Por comparación estilística con las vidrieras del siglo XIV conservadas en la Catedral de Gerona, de hacia 1350-1360, también de un autor desconocido, el llamado Maestro del Presbiterio (2), podemos aventurarnos a afirmar que las vidrieras de Pedralbes y las de Gerona fueron realizadas por el mismo artista, siendo las de Pedralbes un trabajo más temprano. De confirmarse esta teoría, sería importante recordar que éste es el autor normalmente asociado con las famosas tablas de vidriero, utilizadas para la realización de alguna de las vidrieras de la catedral (3).

En noviembre de 1972, el Ayuntamiento de Barcelona se hizo cargo del Monasterio y de la restauración global del

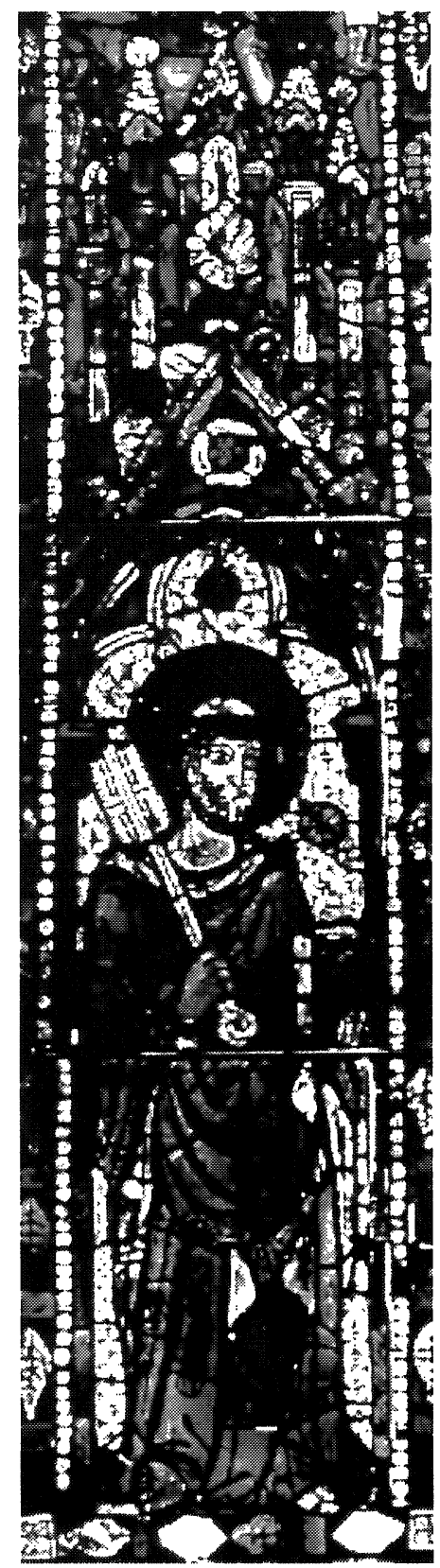

Figura 1.- Vidriera OII. Paneles A1, A2 y A3 antes de la restauración. mismo. Como consecuencia, en septiembre de 1998, una de las vidrieras del ábside de la iglesia, la OII, fue desmontada para proceder a su restauración (4). Esta vidriera está formada por dos lancetas y tracería, con un total de 51 paneles. Las figuras representadas en los paneles inferiores son San Pedro y San Jaime bajo un baldaquino. Los paneles superiores de las lancetas representan motivos geométricos y decorativos mediante una gran variedad de colores (Figura 1). Los paneles de la tracería no representan motivo alguno, siendo meras composiciones abstractas con vidrios de colores. Esta estructura compositiva, repetida en todas las vidrieras del XIV, es también bastante similar a la de las mencionadas vidrieras del Maestro del Presbiterio en la Catedral de Gerona.

Tras el estudio preliminar de los paneles, los hechos más destacados fueron, por un lado, el excelente estado de conservación del vidrio original el cual, con excepción de algunos vidrios rojos plaqué, no presentaba síntomas de corrosión y, por otro, la presencia de una red de plomo medieval en la mayoría de los paneles (5). Por lo que conocemos hasta la fecha, en España, tan sólo las vidrieras del Monasterio de Santes Creus, del siglo XIII, han conservado una red de plomo más antigua que la de Pedralbes (6). Este hecho convierte a los plomos de Pedralbes en un hallazgo de excepcional importancia para la historia del arte de la vidriera española.

\section{LA PRODUCCIÓN DE PLOMOS PARA VIDRIE- RAS DURANTE LA EDAD MEDIA}

El plomo, en forma de varillas, ha sido siempre, desde el comienzo de la producción de vidrieras, el elemento principal utilizado para ensamblar las piezas de vidrio. Las muchas ventajas de este metal, su maleabilidad, flexibilidad, bajo punto de fusión, reducido costo, resistencia a la corrosión, etc., lo han convertido en un material ideal para este propósito, que no ha tenido ningún rival en este campo hasta la introducción del cobre y el hormigón durante este siglo.

Por lo que sabemos, la fuente escrita más antigua sobre la producción y el uso de varillas de plomo para vidrieras sigue siendo hoy en día el manuscrito del monje Theophilus (7), escrito hacia 1120. Theophilus describe detalladamente el proceso de fundido y vertido del plomo en moldes, la elaboración de las varillas de estaño para soldar, el proceso de soldadura, etc. Aparte de Theophilus, el tratado de Antonio da Pisa (8), escrito hacia finales del siglo XIV, es la fuente escrita más contemporánea con respecto a los plomos de Pedralbes. Los procesos de trabajo descritos en estos dos manuscritos, se mantuvieron prácticamente inalterados hasta el siglo XVI y pueden ayudarnos a entender los métodos de trabajo de las vidrieras de Pedralbes. 
Durante la Edad Media, el plomo era vertido en unos moldes de metal o madera compuestos de dos piezas, a fin de obtener las típicas varillas con perfil en forma de $\mathrm{H}$. La superficie de estas varillas, que podían tener una longitud media de unos 40 ó $50 \mathrm{~cm}$, era bastante lisa y regular. Mucho más tarde, en el siglo XVI, se introdujo el uso de los molinillos de plomo para dar forma a las varillas, las cuales empezaron a tener unas longitudes mucho mayores. Este plomo era vertido en moldes y luego estirado y dentado en los molinillos mencionados. La mayor parte de estos primeros molinillos producían sobre el alma del plomo unas muescas o dentaduras las cuales, aparte de facilitar el estirado del plomo con el molinillo, servían para fomentar la adherencia del vidrio al plomo.

Durante la Edad Media, el proceso manual del fundido y vertido del plomo en moldes producía varillas con una gran diversidad de perfiles, mucho más irregulares que las de los plomos posteriores producidos con un molinillo. Los plomos medievales carecen de las esquinas y formas angulosas tan característicos de los plomos actuales. Sus formas son, en general, mucho más suaves y redondeadas, especialmente en las uniones entre el alma y las alas. Estas formas más redondeadas desempeñaban un importante papel en su durabilidad, dándoles una mayor resistencia al deterioro al evitar la tensión acumulada en cualquier zona angulosa.

Sin embargo, las alas de los plomos de Pedralbes son bastante finas y planas, y tienen un grosor algo irregular (0'6-0'9 mm). Las almas tienen, por el contrario, una altura bastante regular ( $3 \mathrm{~mm}$ aproximadamente). Sumando el grosor de las alas, la altura total de los plomos es de unos $5 \mathrm{~mm}$ (9). La anchura media de las alas en una misma varilla de plomo no es muy regular y homogénea, oscilando aproximadamente entre 4 y $6 \mathrm{~mm}$. Esta anchura es, por otro lado, la normal entre los plomos medievales.

El proceso de vertido en moldes producía sobre la parte central de las alas una estructura irregular por el exceso de plomo que sobresalía por las juntas del molde. Tal y como explica Theophilus, a fin de eliminar estas irregularidades, los vidrieros solían raspary alisar las alas de los plomos mediante algún tipo de herramienta metálica afilada en forma de cuchillo. Este proceso era realizado sobre una mesa de trabajo y producía sobre las alas del plomo una estructura a base de líneas de raspado más o menos paralelas a lo largo de las alas. Esto significa que la forma algo redondeada que actualmente vemos en muchos de los perfiles de plomos medievales, era originalmente algo más acentuada. Este proceso de alisamiento no se llevó a cabo en Pedralbes, donde las alas de los plomos son bastante lisas y no muestran este motivo de líneas paralelas.

El siguiente paso era el estirado de las varillas de plomo. Este método, que permitía a los vidrieros obtener una mayor longitud de cada varilla, ahorrando de este modo material, tenía la desventaja de hacer el plomo frágil y quebradizo, incrementando así el riesgo de fracturas. La primera fuente escrita sobre este proceso data aproximadamente de la segunda mitad del siglo XV (10). A finales del siglo XV y principios del XVI, y debido a las desventajas anteriormente mencionadas, esta costumbre fue prohibida por diferentes gremios e Iglesias en Europa Central (11).

Parece lógico suponer que esta costumbre fue utilizada desde el comienzo de la producción de vidrieras, esto es, anteriormente al siglo XV. Así, pues, las mencionadas diferencias en la anchura de las alas podrían inducirnos a pensar que este proceso de estirado de las varillas fue utilizado en Pedralbes. No obstante, si bien pensamos que estas diferencias de anchura, son más bien debidas a las formas irregulares de los moldes en los cuales se vertía el plomo, dada la gran carencia de información que existe sobre este aspecto concreto, sería necesario un estudio mucho más profundo a fin de poder contestar a estas preguntas.

Una vez cortado el vidrio y pintadas las piezas principales, los vidrieros procedían al emplomado de las piezas de vidrio. En general, podemos afirmar que el proceso de emplomado era realizado prácticamente de la misma manera a como se hace hoy en día, esto es, insertando los vidrios en las varillas de plomo, introduciendo los extremos de una o varias varillas bajo las alas otra principal y creando de esta manera un punto de soldadura. En este punto es interesante destacar que las varillas de plomo de Pedralbes, a la altura de los puntos de soldadura, no están insertadas una dentro de otra sino más bien en contacto superficial entre sí. Esto es, no existe un contacto directo entre las almas de las varillas, lo que origina un espacio de unos 2-3 mm entre ellas. Si bien este espacio no es visible al estar los puntos de soldadura cubiertos superficialmente por el estaño, este método de trabajo produce unos puntos de soldadura muy débiles, lo que puede afectar seriamente a la estabilidad de los paneles, como se puede apreciar en zonas puntuales.

Según Theophilus, el estaño utilizado para soldar era fundido y luego vertido en los mismos moldes utilizados para las varillas de plomo. De esta manera era posible obtener finas varillas de estaño y se evitaba el tener que hacer unos moldes especiales para el estaño.

Tal y como ya explica Theophilus, antes de proceder a la soldadura de los paneles, cada zona de unión entre dos o más varillas era ligeramente raspada a fin de eliminar tanto las irregularidades del plomo como la capa de oxidación, que impide una buena adherencia entre el estaño y el plomo. A continuación se aplicaba algún tipo de grasa o aceite sobre esos puntos, el cual facilitaba, asimismo, la adherencia entre el plomo y el estaño. Las 
soldaduras eran realizadas con una barra de hierro incandescente. Se aplicaba una pequeña porción de estaño a la punta del soldador, el cual se apoyaba sobre el punto de soldadura durante unos segundos, hasta que el estaño se hubiera extendido por el plomo y adherido a él.

Durante este proceso, los vidrieros, tan sólo mediante la experiencia e intuición personal, debían tener un cuidado especial en mantener la temperatura de la barra de hierro utilizada para soldar por encima de los $183^{\circ} \mathrm{C}$ (punto de fusión del estaño de soldar) y por debajo de los $327,4^{\circ} \mathrm{C}$ (punto de fusión del plomo), a fin de no quemar el plomo (12). La evidente dificultad que planteaba el control de la temperatura durante esta operación es patente en algunas zonas de plomo quemado y en las formas caprichosas que presentan los puntos de soldadura en muchas redes de plomo medievales. En los paneles de Pedralbes no hemos hallado estas zonas de plomo quemado, si bien las formas irregulares del estaño en estos puntos probablemente indican que la temperatura de la barra de soldar era demasiado elevada y el tiempo de contacto entre la barra de soldar y la varilla fue demasiado largo.

El grosor de los puntos de soldadura en Pedralbes oscila entre 1 y $2 \mathrm{~mm}$ y éstos son bastante largos en extensión. Forman unos pegotes gruesos e irregulares de estaño, que se extienden unos 2 ó $3 \mathrm{~cm}$ fuera del punto de soldadura a lo largo de las varillas de plomo. En muchas zonas, incluso sobrepasan el perímetro de las alas o las cubren tan sólo parcialmente, también en forma de pegotes gruesos y apuntados. La cantidad de estaño utilizada en estos puntos es evidentemente excesiva, tanto en grosor como en extensión. Muchos de estos puntos de soldadura están situados en medio de una varilla sobrepasándola por los laterales a fin de cubrir los huecos creados por las piezas de vidrio que no encajan en las varillas (Figura 2). En Pedralbes, todos los puntos de soldadura son contemporáneos a la red de plomo, esto es, no existen soldaduras posteriores.

Normalmente, los paneles eran soldados por ambas caras, tal y como describe Theophilus y podemos vemos en Pedralbes. El hecho de que se recomiende esta práctica significa que no siempre era llevada a cabo, dejándose una de las dos caras sin soldar a fin de abaratar costos. A veces, los puntos de soldadura en los plomos laterales se soldaban tan sólo por una cara a fin de facilitar su eliminación en caso de que los paneles, una vez fueran a ser instalados, no encajaran en las ranuras de los marcos de piedra (maineles y tracería). Esta práctica fue llevada a cabo en Pedralbes, donde los puntos de soldadura de la cara exterior en los plomos laterales se dejaron sin soldar.
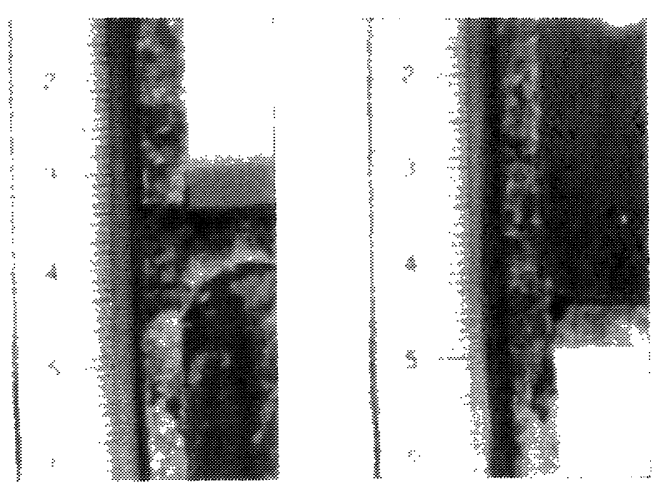

$x$
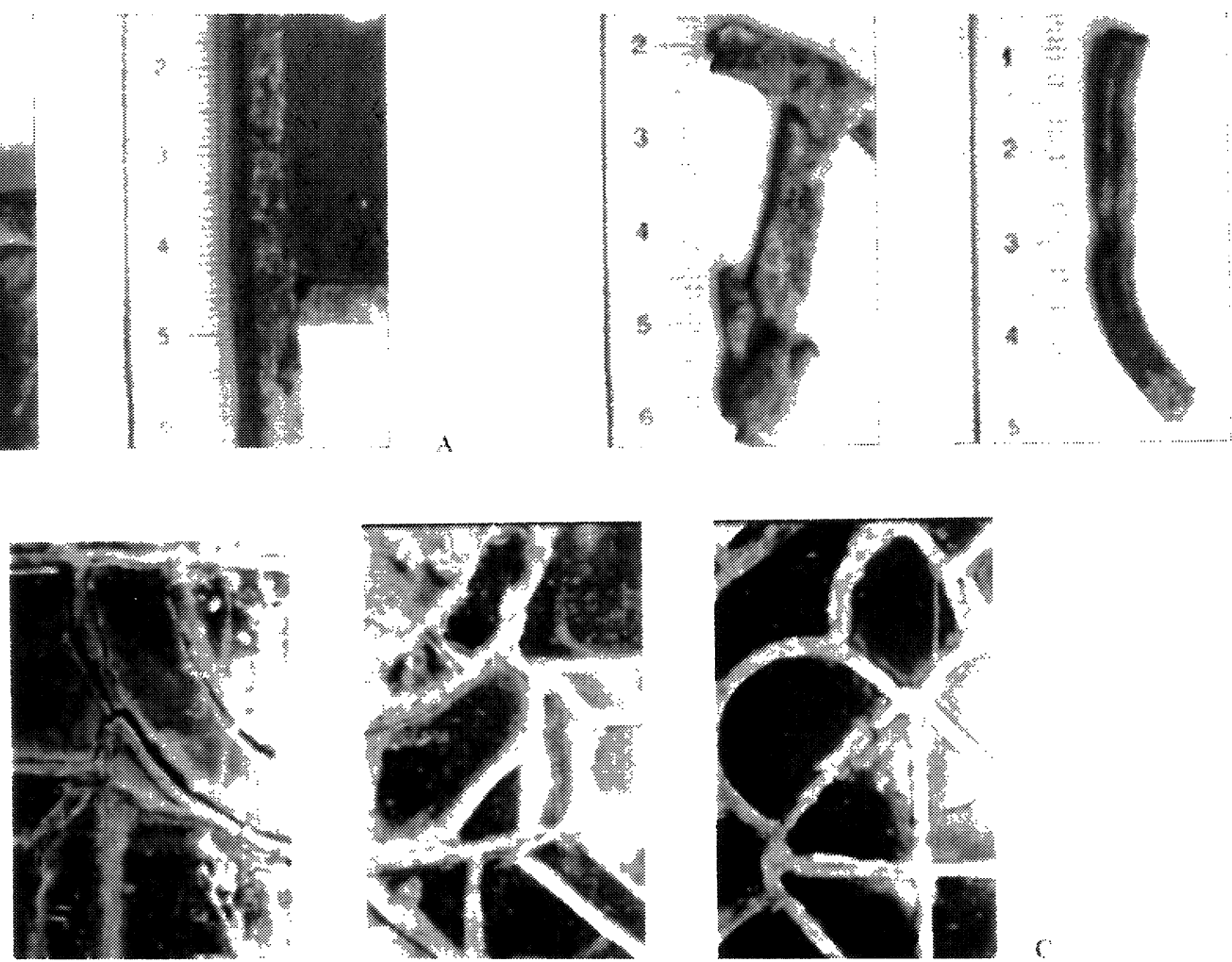

(

Figura 2.- Detalle de la unión entre dos plomos. Con soldadura (cara int., foto izq.) y sin soldadura (cara ext., foto der.) (A); plomos ciegos con soldadura (foto izq.) y plomo de sujeción de las barras de refuerzo (foto der.). Ambos de fecha posterior (B); distintos detalles de la red de plomo medieval por la cara exterior $(\mathrm{C})$. 
Una práctica común entre algunos vidrieros durante la Edad Media era la inserción de una o dos barras de metal o madera entre dos o tres plomos (13). Estas barras, que podían tener un trazado recto o curvo, tenían dos funciones principales. La primera era el refuerzo de los paneles, a fin de darles mayor estabilidad y resistencia. La segunda era una función estética, donde los diferentes grosores en la red de plomo eran utilizados con un carácter estético y decorativo (14). Estas varillas dobles solían estar completamente estañadas por ambas caras a fin de cerrar el hueco existente entre ambas. En los paneles de Pedralbes no se utilizó esta técnica y en España, por lo que sabemos, no se ha conservado ningún ejemplar con esta técnica, si es que alguna vez fue utilizada (15).

Otra práctica habitual entre los vidrieros era el estañado de toda la superficie exterior de las varillas de plomo por una o por las dos caras, a fin de proporcionar a los paneles mayor estabilidad. Algunos autores han apuntado la posibilidad de que este estañado completo de la red de plomo tuviera también una función estética, aunque esta teoría carece de fundamento dada la altura de las vidrieras y el hecho de que este estañado no es visible cuando la luz atraviesa los paneles. Sea cual fuera el motivo de su utilización, esta técnica no fue utilizada ni Pedralbes ni en ninguna de las otras redes de plomo medievales conservadas en España.

En ocasiones los vidrieros solían grabar ciertas marcas o cifras sobre las alas de los plomos en los laterales de los paneles. Estos signos, que podían ser en forma de círculos o cifras romanas, indicaban la posición de cada panel dentro de un vano concreto en la vidriera o incluso en el edificio y facilitaban a los vidrieros la rápida localización de los paneles a la hora de proceder al montaje de las vidrieras. Según Frenzel (16), esta práctica desapareció gradualmente hacia finales del siglo XIV, o al menos no se ha hallado ejemplar alguno posterior a esta fecha. Tampoco en este caso hemos encontrado en Pedralbes algún tipo de marca o inscripción similar sobre el plomo.

Normalmente, uno de los pasos finales en la creación de una vidriera es el enmasillado de los paneles. No obstante, en ningún tratado o documento anterior al siglo XVI encontramos referencia alguna sobre el uso de masilla $u$ otro material de cerramiento similar. El uso de la masilla, utilizada para asegurar las piezas de vidrio a la red de plomo, dar mayor estabilidad a los paneles e impedir el paso del agua de lluvia y el viento, fue probablemente introducido durante el siglo XVI, época de la que datan los documentos más antiguos conservados (17). Los paneles de Pedralbes no fueron enmasillados durante la última restauración y parece bastante probable que tampoco lo fueran originalmente. Un hecho que apoya esta teoría es la presencia de grandes pegotes de estaño de soldar utilizados para cubrir los huecos dejados por vidrios demasiado pequeños, los cuales son normalmente cubiertos con masilla.

El último paso antes de la instalación de los paneles era la colocación de dos o tres barras de sujeción en hierro sobre los paneles. Estas barras sueltas podian ser horizontales, verticales o curvas y se sujetaban a los paneles mediante unas pequeñas tiras o nudos de plomo soldadas a la cara interior, exterior o ambas caras de los paneles (18). Otra variante es la de algunas de las vidrieras medievales de la Catedral de Altenberg, donde encontramos en la cara interior de los paneles, una barra horizontal con una pequeña curvatura donde se encaja otra barra vertical. Cada uno de los paneles rectangulares de Pedralbes está reforzado con dos barras horizontales de hierro soldadas por la cara interior. Estas barras, si bien no parecen ser las originales, son bastante antiguas. Aunque en los documentos medievales conocidos no existe prácticamente referencia alguna al uso de estas barras (19), el hecho de que un gran número de estas barras se hayan conservado en vidrieras del siglo XIII y XIV, probablemente nos indica que ésta era una práctica común durante la Edad Media, por lo que es bastante probable que también los paneles de Pedralbes fueran originalmente reforzados con estas barras.

\section{DATACIÓN DE LA RED DE PLOMO DE PEDRALBES}

Dada la numerosa presencia de plomos de fractura, podemos deducir que la red de plomo en los paneles de Pedralbes no es la original. Estos plomos de fractura, si bien no forman parte de la composición original, son contemporáneos a la red de plomo actual. El desemplomado y nuevo reemplomado de los paneles era una práctica habitual entre los vidrieros, incluso al poco tiempo de la instalación de la vidriera, debido a diferentes causas. Las fracturas en el vidrio solían repararse mediante la introducción de un nuevo plomo, normalmente algo más estrecho que los ya existentes. Si bien estos plomos servían para recuperar la pieza fracturada, los problemas que presenta su uso son varios. Por un lado, creaban una lectura distorsionada del dibujo de la red de plomo y suponían un impedimento para la correcta lectura de la composición y por otro lado, para poder introducir el plomo de fractura era necesario eliminar unos $2 \mathrm{~cm} \mathrm{del}$ vidrio original a lo largo de la línea de fractura.

Por lo que respecta a la cantidad total de plomo medieval conservado en estas vidrieras de Pedralbes, podemos afirmar que aproximadamente un $98 \%$ de la red de plomo de los paneles es del siglo XIV. Este $2 \%$ de plomo de otros períodos está justificado por la presencia de algunas varillas posteriores, posiblemente del siglo XVI, añadidas a los laterales de algunos paneles, algunos plomos de fractura superficiales, esto es, sin alma, soldados 
por ambas caras de las fracturas; las tiras o nudos de plomo utilizados para sujetar las barras de refuerzo; y algún plomo, muy escasos, con dentaduras. Estos cuatro tipos de plomos son posteriores a la red de plomo, siendo los tres últimos probablemente del siglo XVII ó XVIII. Sigue pendiente el poder datar la red de plomo de estos paneles. Para ello, hemos tenido en cuenta diferentes factores.

En primer lugar, el hecho de que las varillas de plomo han sido fundidas y vertidas en moldes. Esta teoría viene corroborada por la forma irregular de las varillas y por la ausencia de dentaduras en el alma de las varillas, las cuales empezaron a aparecer en el siglo XVI por la utilización de los molinillos de plomo.

En segundo lugar, según la clasificación de plomos medievales realizada por Frenzel (20), el plomo de Pedralbes coincidiría con la categoría 3 (principios del siglo XIV) ó 7 (1380-1550). Aunque esta clasificación no debería ser tomada de forma literal, ofrece al menos una datación aproximativa de gran utilidad.

Tomando en consideración estos factores, sería bastante probable que la red de plomo de Pedralbes datase de la segunda mitad del siglo XIV.

\section{ESTADO DE CONSERVACIÓN DEL PLOMO DE PEDRALBES}

En líneas generales, podemos afirmar que el plomo de Pedralbes es, en comparación con los plomos actuales, bastante más duro y mucho menos flexible. En su superficie se aprecia una pérdida de brillo y oscurecimiento del metal debido a la formación de una capa de oxidación. Esta capa de oxidación está acompañada por unas gruesas capas de residuos y deposiciones de óxidos del metal que cubren toda la superficie exterior del plomo y tienen una coloración marrón clara. No se aprecian, sin embargo, fenómenos de corrosión evidentes, ni en el propio plomo ni en el estaño, como sucede con otros plomos medievales conservados en otras vidrieras europeas.

En otras zonas hemos podido distinguir diferentes estados de fracturas en la zona de unión entre el estaño y el plomo y sobre el plomo mismo. Las primeras están relacionadas con el método utilizado para soldar los paneles, el cual desempeña un papel muy importante en la estabilidad y conservación de las vidrieras. Las segundas, con las presión del viento y consiguiente abombamiento de los paneles. Por lo que respecta a las fracturas en la zona de unión entre el estaño y el plomo, conviene destacar dos hechos importantes: por un lado, la cantidad de estaño aplicada sobre el plomo y por otro lado, la tensión que se produce entre un metal frío (plomo) y otro caliente (barra de soldar) durante el soldado de los paneles. Como consecuencia, demasiado poco estaño produ- ce debilidad en el metal y demasiado estaño puede originar fracturas alrededor de los puntos de soldadura, debido a los diferentes coeficientes de expansión del plomo y el estaño. Por otro lado, le tensión creada entre dos metales con temperaturas muy diferentes acelera la fatiga del plomo, lo que puede originar, asimismo, fracturas en las zonas de contacto entre el plomo y el estaño.

Como ya se ha mencionado, los paneles de Pedralbes muestran una gran deformación o abombamiento de la red de plomo hacia la cara interior, especialmente en la parte inferior de los mismos, debido a la presión del viento, vibraciones, ciclos térmicos y fatiga natural que afecta al plomo con el paso de los años. Este fenómeno no es exclusivo de los plomos medievales. Así pues, muchas vidrieras con plomo de este siglo también muestran estas típicas deformaciones. Estas deformaciones dificultan el manejo de los paneles durante su restauración y han provocado fracturas en las varillas de plomo y la salida de algunas piezas de vidrio de las varillas de plomo.

Algunos plomos en los bordes laterales de los paneles se han perdido, lo cual ha hecho necesario, durante la restauración actual, el uso de nuevas varillas de plomo para integrar esas lagunas.

\section{COMPOSICIÓN QUÍMICA GENERAL DE LOS PLOMOS}

El plomo en su estado natural contiene toda una serie de impurezas, que ya eran conocidas desde muy antiguo. La eliminación de estas impurezas también era practicada desde muy antiguo, tal y como atestiguan los análisis de plomos egipcios y romanos. No obstante, en ninguno de los tratados mencionados se hace mención alguna al proceso de eliminación de estos componentes menores del plomo. Hoy en día sabemos que la producción de plomo con una pureza por encima de un $99 \%$ ya había sido lograda por los romanos y es este plomo, relativamente puro, el que se utilizó en las vidrieras durante la Edad media (21).

Hoy en día sabemos que el plomo medieval tiene una composición ligeramente distinta a la del plomo producido después de 1833 , fecha en la que se perfeccionó el sistema de eliminación de impurezas, especialmente de plata. A pesar de este proceso de depuración, el plomo medieval seguía conteniendo ciertas impurezas menores, tales como plata, estaño, cobre, antimonio, etc. Estas ligeras diferencias de composición entre el plomo medieval y el plomo actual son debidas a varios motivos: el pequeño porcentaje de impurezas que permanecían en el plomo tras la primera depuración; las impurezas accidentalmente recogidas por el plomo durante su fabricación; y el proceso de reciclaje, y refundido de plomos antiguos procedentes de otras vidrieras, para ser reutilizados en nuevas vidrieras, los cuales, al no haberse eliminado 
previamente los puntos de soldadura, añadían más estaño a la composición.

El estudio microscópico del plomo puede ofrecernos, asimismo, una valiosa información sobre su estructura granular interna, el proceso de recristalización, la presencia de impurezas, el método de trabajo, etc. La estructura interna del plomo está formada por cristales, entre los cuales pueden distinguirse los diferentes elementos de traza o componentes menores del plomo, dado que éstos no llegan a fundirse entre sí. El grosor de estos cristales varía en función de la composición del plomo y, por lo tanto, de su datación. En líneas generales, el plomo "puro" producido a partir del siglo XIX, está compuesto por cristales más grandes (diámetro medio de $77 \mu \mathrm{m}$ ) que el plomo medieval (diámetro medio entre 10 y $30 \mu \mathrm{m}$ ), dada la mayor cantidad de impurezas que este último contiene.

Por lo que respecta al estaño de soldar, éste estaba constituido básicamente por una aleación de plomo y estaño. Hoy en día, el estaño utilizado para soldar los plomos de las vidrieras contiene un $61,9 \%$ de estaño y un $38,1 \%$ de plomo y tiene un punto de fusión de $183^{\circ} \mathrm{C}$. Históricamente, las proporciones de estos dos componentes podían variar enormemente de un vidriero a otro. Estas diferencias son también evidentes en los dos tratados mencionados. Así pues, mientras que Theophilus recomienda el uso de 1 parte de plomo y 5 partes de estaño (16,6\% / 83,3\% respectivamente), Antonio da Pisa aconseja 1 parte de plomo y 1 parte de estaño (50\% $150 \%$ respectivamente). Es importante destacar que estas diferencias de composición podían también desempeñar un papel importante en la estabilidad y el estado de conservación de las vidrieras.

\section{EL DETERIORO DEL PLOMO MEDIEVAL}

Aunque no es la intención de este estudio el ofrecer una descripción detallada de los distintos factores y procesos de deterioro del plomo, quisiéramos destacar algunos de los aspectos más importantes a fin de tener una imagen más clara del estado de conservación de las vidrieras de Pedralbes.

La formación de una película protectora de oxidación y su resistencia a la corrosión en diferentes medios (atmosférico, agua del mar, productos químicos, etc.) son dos de las propiedades más conocidas del plomo. No obstante, existen toda una serie de factores que pueden acelerar su pérdida de resistencia al deterioro, fomentando el avance de distintos fenómenos de oxidación y corrosión. Estos factores pueden ser internos, como la composición del plomo, o externos, como la presencia de agua y contaminantes ambientales.

Por lo que respecta a la composición del plomo, los pequeños porcentajes de impurezas contenidas en el plomo medieval, lo hacen bastante diferente en cuanto a su resistencia al deterioro y durabilidad. El plomo prácticamente puro obtenido a partir de 1833 , suele mostrar mayores síntomas de fatiga y fracturas, fenómeno éste que no es tan corriente en los plomos anteriores a esta fecha (22). Un elevado porcentaje de estaño, antimonio o cobre aumenta considerablemente la resistencia a la tensión y la dureza del plomo a temperatura ambiente, haciéndolo más rígido y menos elástico, pero también más resistente a la corrosión. Por otro lado, el cobre y la plata alteran la estructura cristalina del plomo, reduciendo considerablemente el tamaño de los cristales de la red y le confieren mejores propiedades mecánicas, permitiéndole una mayor flexibilidad y resistencia a las tensiones mecánicas.

Asimismo, la presencia de fracturas en el plomo puede fomentar el avance de la corrosión. Estas fracturas, originadas por tensiones mecánicas, van avanzando hacia el interior del plomo por entre los cristales, los cuales se van separando sin ser fracturados. Estas aberturas facilitan la entrada del agua contaminada, la cual puede estimular el avance de los procesos de corrosión, desintegrando gradualmente la estructura granular.

Los principales factores externos son las altas humedades, ciertos gases presentes en la atmósfera, como el dióxido de carbono o el dióxido de azufre, algunos ácidos, como el ácido acético y el ácido nítrico, algunas especies de maderas, sobre todo en un medio húmedo, algunos productos disociados del azufre y el cloro, ciertas pinturas comerciales, ciertos productos de limpieza, etc. (23).

Por lo que respecta a las condiciones ambientales, la presencia de agua es un factor esencial para el avance de la corrosión del plomo. Como hemos mencionado, algunos gases contaminantes presentes en el agua de lluvia o de condensación pueden acelerar este proceso. Estos gases determinan el nivel de acidez del agua. Los dos principales tipos de contaminantes son los gaseosos $\left(\mathrm{SO}_{2}, \mathrm{No}_{x}, \mathrm{O}_{3}, \mathrm{Cl}_{2}, \mathrm{HCl}, \mathrm{H}_{2} \mathrm{~S}\right.$, ácidos orgánicos, etc.) y las partículas de aerosoles (cloruros, sulfatos, nitratos, etc.) (24).

El primer paso en este proceso de deterioro es la formación de una película protectora de óxido o carbonato de plomo, fuertemente adherida a la superficie. Esta película se forma mediante una rápida reacción entre el plomo y la atmósfera. Esta primera reacción va seguida de una segunda, entre esta película de oxidación y el dióxido de carbono presente en la atmósfera, lo que da lugar a la formación de una segunda película protectora de carbonato de plomo. Estas películas evitan el posterior contacto del plomo puro con la atmósfera, impidiendo el avance de la corrosión.

Los productos de reacción formados por el dióxido de carbono $\left(\mathrm{CO}_{2}\right)$ presente en el agua con los iones del 
plomo $\left(\mathrm{Pb}^{2+}\right)$, pueden ser de dos tipos: carbonatos neutrales o básicos. Estos productos son muy insolubles y forman una capa homogénea de sales inorgánicas que contribuyen a la protección del plomo. Asimismo, mediante el contacto con ácido sulfúrico se produce la formación de una capa similar de sulfato de plomo muy adherente, la cual es insoluble a la mayoría de ácidos y protege el plomo de un ulterior ataque.

Por el contrario, la combinación del los iones del plomo con especies orgánicas, como el ácido fórmico, acético, oxálico, etc., puede disolver estos productos, haciendo que el plomo pierda su resistencia a la corrosión. Este proceso se ve acelerado en un medio húmedo.

Otros factores externos que pueden disolver la mencionada película superficial de oxidación, fomentando así el progresivo avance de la corrosión del plomo, son las disoluciones de ácidos orgánicos procedentes de maderas húmedas. Asimismo, el cemento Portland contiene álcalis libres que pueden también estimular un ataque lento del plomo en una atmósfera húmeda. Por último, otro tipo de corrosión, la electrolítica, puede originarse cuando el plomo se halla en contacto directo con otro metal distinto, creándose, de esta manera, una reacción galvánica.

\section{ANÁLISIS CON PRUEBAS ORIGINALES}

Dentro de este proyecto de investigación, se estudiaron tres muestras del plomo de Pedralbes en el Bundesanstalt für Materialforschung und-prüfung en Berlín por el Dr. Detlef Kruschke (Figura 3). Las muestras fueron examinadas bajo un microscopio con aumentos entre $25 \mathrm{x}$ y 500x. Para la realización de este estudio se tomaron en cuenta diferentes aspectos: el plomo, el estaño, las fracturas, los fenómenos de oxidación y la presencia de elementos traza. El análisis de las muestras se realizó mediante una microsonda. Con esta técnica, tan sólo las concentraciónes de plomo superiores a un $99 \%$ y los elementos de traza con una concentración superior a un $0,1 \%$ pudieron ser detectadas.

El proceso de trabajo fue el siguiente: una pequeña parte representativa de cada muestra, de unos $5 \mathrm{~mm}$ aproximadamente, fue introducida en una resina epoxi, siendo a continuación pulida. Las zonas pulidas fueron brevemente atacadas (5-10 segundos) con una disolución de 4,25 partes de glicerina, 1 parte de ácido nítrico, y 1 parte de ácido acético, siendo luego aclaradas con agua y fotografiadas bajo el microscopio. Se intentó un segundo ataque con una disolución de 2 partes de etanol, 0,24 partes de ácido clorhídrico y 0,12 partes de ácido nítrico

Pedralbes, Muestra 1: sección transversal de una varilla de plomo a la altura de un punto de soldadura relativamen- te grueso. Los cristales de color obscuro representan el plomo y los de color claro el estaño. En la muestra se puede apreciar claramente una estructura interna a lo largo del alma del plomo originada durante el proceso de vertido en el molde y posterior enfriamiento. A lo largo de esta estructura se puede reconocer una mayor presencia de elementos de traza, especialmente estaño, posiblemente producida por una temperatura demasiado elevada de la barra de soldar. No se aprecia ningún fenómeno de corrosión. El diámetro medio de los cristales es de $19,9 \mu \mathrm{m}$.

Pedralbes, Muestra 2: sección transversal de una tira de plomo utilizada como nudo para sujetar las barras de transversales de hierro. Los cristales de color obscuro representan el plomo y los de color claro el estaño. En esta muestra tampoco se pueden apreciar fenómenos de corrosión. El diámetro medio de los cristales es de $25,2 \mu \mathrm{m}$.

Pedralbes, Muestra 3: sección transversal de una varilla de plomo a la altura de un punto de soldadura relativamente grueso. Este plomo, dada la presencia de marcas de molinillo (dentaduras), procede de alguna restauración posterior, probablemente del siglo XVII ó XVIII y es un plomo estirado. Su perfil es distinto al de los plomos medievales (zonas angulosas y alas muy finas). El diámetro medio de los cristales es de $17,8 \mu \mathrm{m}$.

\section{Resultados de los análisis:}

Tanto en los plomos medievales como en los otros plomos posteriores analizados, no se aprecia presencia alguna de corrosión. El buen estado de conservación del plomo medieval es probablemente debido a las impurezas o elementos de traza menores presentes en su composición, a la gruesa capa protectora de oxidación que cubre toda su superficie y a unas condiciones atmosféricas favorables en el interior y exterior del monasterio.

\section{CONCLUSIONES}

Para concluir quisiéramos destacar brevemente los resultados más destacados de este estudio. En primer lugar, la gran importancia de esta red de plomo medieval para el estudio de la vidriera española y las técnicas de trabajo de la Edad media. En segundo lugar, su buen estado de conservación. En tercer lugar, la inferior calidad del trabajo manual y el poco cuidado por los detalles de acabado y producción visibles en esta red de plomo en comparación con otras conservadas en Europa.

La explicación de esta inferior calidad puede estar relacionada con el método de trabajo de un autor concreto o con la menor tradición que ha existido históricamente en España en el campo de las vidrieras, en comparación con aquellos países donde este arte se desarrolló, 

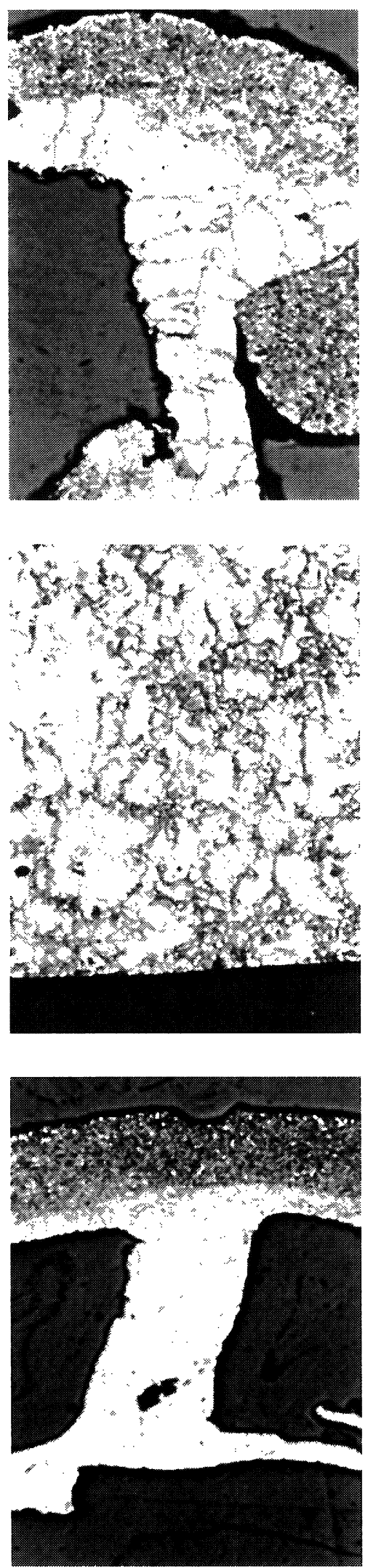

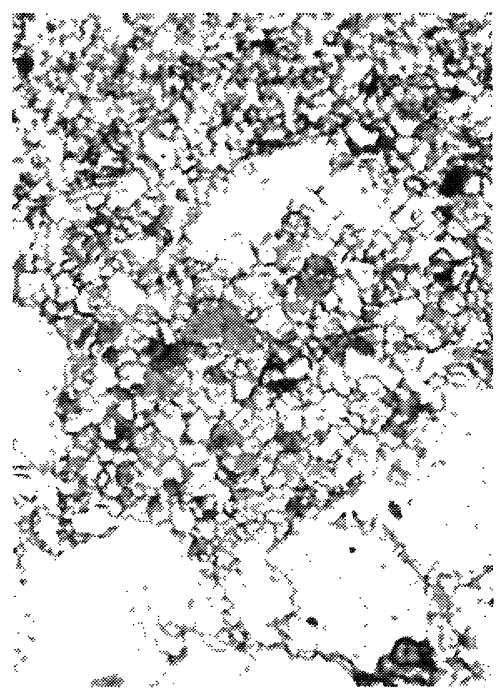

Muestra 1

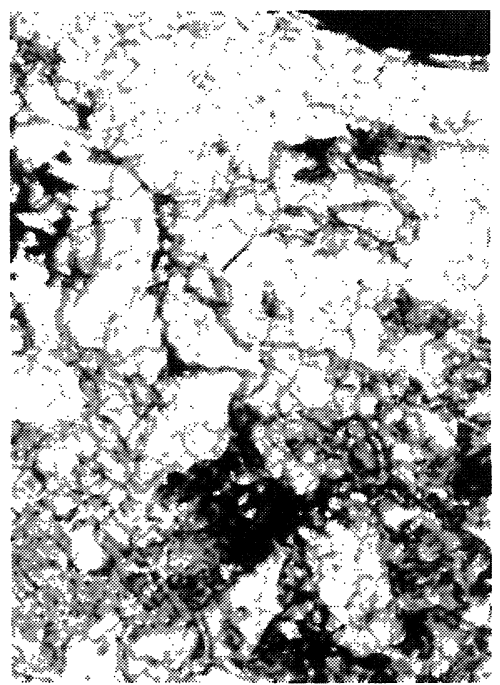

Muestra 2

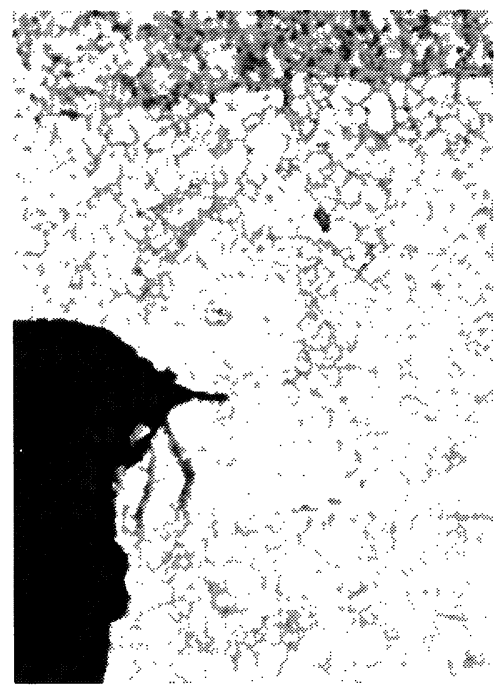

Muestra 3

Figura 3.- Corte transversal de un plomo medieval, donde se aprecia la estructura vertical producida durante el vertido y enfriado del plomo y un gran punto de soldadura (25x), Muestra 1 (izq:); detalle microscópico del mismo plomo medieval donde se aprecia la presencia de estaño en su estructura cristalina (10)x). Muestra 1 (der.). Detalle microscópico de la estructura cristalina de un plomo sin alas utilizado para sujetar las barras transversales de refuerzo ( $100 \mathrm{x}$ ). Muestra 2 (izq.); detalle microscópico de la estructura cristalina del mismo plomo, donde se aprecia la presencia de estaño (100x), Muestra 2 (der.). (orte transversal de un plomo del siglo XVII ó XVIII con alas mucho más estrechas que el alma y una gran capa de estaño (25x), Mucstra 3 (izq.); detalle de la estructura cristalina del plomo y presencia de fisuras entre el alma y las alas (100x), Muestra 3 (der.). 
especialmente Alemania, Francia e Inglaterra y Austria. En estos países, el mayor desarrollo de este campo en todos los sentidos sigue siendo, incluso hoy en día, bastante más evidente.

No obstante, esta baja calidad en el trabajo manual contrasta con el sorprendente buen estado de conservación del plomo. Éste es debido a su composición química, a las favorables condiciones climáticas del exterior e interior de la iglesia del monasterio y a las afortunadamente escasas restauraciones efectuadas en las vidrieras.

\section{AGRADECIMIENTOS}

Quisiera expresar mi agradecimiento a aquellas personas que, de alguna u otra manera han contribuido en este trabajo de investigación.

-Glasmalerei Peters, Paderborn (Alemania), por proporcionarme todo tipo de facilidades para realizar este trabajo.

-El Servei d'Arqueologia Urbana (M.H.C.B.) del Ayuntamiento de Barcelona, por las muestras de materiales originales facilitadas.

-Dr.Detlef Kruschke del Bundesanstalt für Materialforschung und -prüfung, Berlín, por realizar los análisis de las muestras y ayudarme a interpretar los resultados.

-Isora Díaz Rodríguez, de Glasmalerei Peters, Paderborn (Alemania), por la realización de los dibujos técnicos del plomo y la lectura y corrección del texto.

-Dra. Nieves Valentín, del Instituto del Patrimonio Histórico Español y el C.S.I.C., Sección Biología, Madrid, por la corrección final del texto.

-Dr. Domingo Gimeno Torrente, del Departamento de Geoquímica de la Facultad de Geología en la Universidad de Barcelona.

-Antoni Vila Delclós, del C.V.M.A. Cataluña, por la información facilitada sobre otros plomos medievales en Cataluña.

\section{NOTAS RESEÑADAS EN EL TEXTO}

(1) Aparte de estas vidrieras del siglo XIV, el Monasterio posee también otras del siglo XVIII y algunos fragmentos de los siglos XV y XVI. Ainaud de Lasarte, J., Vila-Grau, J., Escudero i Ribot, M. A., Vila i Delclós, A., Cañellas, S., Mundo, A. M. Els vitralls de la catedral de Barcelona i del Monestir de Pedralbes. CVMA, Espanya 9, Catalunya 4. Institut d'Estudis Catalans. Barcelona, 1997, pp. 26.

(2) Ainaud de Lasarte, J., Vila-Grau, J., Assumpta Escudero i Ribot, M., Vila i Delclós, A., Marques, J., Roura, G, Marque ., J.ME,ls vitralls de la Catedral de Girona. CVMA, Espanya 7, Catalunya 2. Institut d'Estudis Catalans. Barcelona, 1987, pp. 17-19.

(3) Estas dos tablas de vidriero se encuentran actualmente expuestas en el Museo de la Catedral de Gerona.

(4) Los trabajos de restauración están siendo realizados en la Secció d'arqueología Urbana del Ayuntamiento de Barcelona.

(5)Tan sólo dos paneles han sido reemplomados durante la última restauración, probablemente a finales del siglo XIX (CVMA, Espanya 9, Catalunya 4).

(6) Aparte de estos dos casos mencionados, tan sólo en la Parroquia de Santa María de Cervera y en las catedrales de Gerona y Tarragona se han conservado vidrieras o pancles con plomo medieval, si bien algo posterior a éstos.

(7) Theophilus, De Diversis Artibus.

(8) Pisa, A. da, Arte Delle Vetrate.

(9) Es importante destacar en este punto que la altura del alma de los plomos desempeña un papel más importante que su anchura de cara a la estabilidad final de los paneles.

(10) Frenzel, G. (1991), pp. 2-3.

(11) Esta prohibición la encontramos en los estatutos de varios gremios(Wismar -fecha desconocida-, Rostock -1476-, Freiburg -1484 y 1513 y Praga -1527-) y en los documentos de algunas iglesias (St. Adalbert en Breslau -repetidas ocasiones entre 1487 y $1500-$ ). Información procedente de Oidtmann, H. (1929), pp. 451-452 y Frenzel, G. (1991), p. 2.

(12) El punto de fusión del plomo $\left(327,4^{\circ} \mathrm{C}\right)$ se ve considerablemente reducido por la adición del estaño $\left(232^{\circ} \mathrm{C}\right)$. Esta nueva aleación tiene un punto de fusión de $183^{\circ} \mathrm{C}$, mucho más favorable para soldar sobre el plomo.

(13) Las barras de metal eran de hierro y podían tener un perfil circular o rectangular de unos 4-5 mm. Para las barras de madera se utilizaba junco fresco con un diámetro de unos $3 \mathrm{~mm}$. En ocasiones, como en las vidrieras de ábside de la Catedral de Colonia, se utilizaron en un mismo panel tanto barras de madera como de hierro.

(14) La utilización de dos varillas de plomo para reforzar ciertas líneas del dibujo, producía la impresión de plomos mucho más anchos. De estas diferencias de grosor, entre las líneas principales del dibujo y las secundarias, podemos deducir el importante papel estético que la red de plomo desempeñaba como elemento de diseño en la composición general durante la Edad media.

(15)Según Oidtmann,H. (1912), pp. 40-41, este procedimiento era característico de los países germánicos, donde se ha encontrado una mayor cantidad de ejemplares de este tipo en vidrieras medievales (Colonia, Altenberg, Haina, Kappenberg, Roager, Lindena, Wimpfen, Wetzlar, Limburg, etc.). No obstante, también en Austria (Stiftskirche Heiligkreuz), Francia (Catedral de Estrasburgo), Suecia (Gotland), etc., consta la presencia de estas barras de refuerzo.

(16) Frenzel, G. (1991), p. 2.

(17) En este sentido, el famoso documento de 1531 procedente del libro de cuentas de la Catedral de Salisbury (Reino Unido), es la primera referencia conocida sobre el uso de masilla ("sement") en las vidrieras (Smith, J. T., Antiquities of Westminster. Londres, 1807).

(18) La mayoría de las barras medievales conservadas tienen una sección circular o rectangular de aproximadamente $1 \mathrm{~cm}$ de diámetro.

(19) En el Capítulo XIX del Tratado de Antonio da Pisa, podría quizá hacerse referencia, si bien de forma no muy clara, al uso de cstas barras. 
Por otro lado, en un documento medieval (sin fecha concreta) hallado en Westminster (Reino Unido), se menciona claramente su uso: "For small bars of iron, called sondlets, to hold the glass in the said windows"(Smith, 1807).

(20) Frenzel, G. (1991), p. 4.

(21) Conviene aclarar que el proceso de la eliminación de impurezas en el plomo durante la Edad media no era llevado a cabo por los vidrieros.

Éstos compraban las barras de plomo ya depurado y las volvían a fundir y vertir en moldes para así fabricar las varillas.

(22) Newton, R. G. (1996), p. 143.

(23) Noehl, R. (1991), p. 26.

(24) Fiaud. C. (1993), p. 170

\section{BIBLIOGRAFÍA DE INTERÉS SOBRE EL PLOMO EN VIDRIERAS}

Bauer, W. P., Reihenuntersuchung von Verbleiungstücken Mittelalterlicher Glasgemälde verschiedener Provenienz, en "Ö.Z.K.D.", 21 (1967), pp. 207-209.

Brown, S. L., The Structure of Lead as Related to Stained Glass, en "Journal of the British Society of Master Glass Painters" Vol. II, $\mathrm{n}^{\circ} 3(1928)$, pp. 123-128.

Cannon, L., Lead Milling Marks from a Sixteenth Century Window, en "Stained Glass Quarterly", Fall, 1988, pp. 222-226.

Cannon, L. y Goldkuhle, D., A study of the Physical and Chemical Properties of Lead Calme and the Deterioration and Stability of Leades Stained Glass, en Comisión del CVMA en Suiza, Tagung für Glasmalereiforschung, Ponencias del XVI Coloquio Internacional, Berna, 1991.

Cortés F., Medieval window leads from the Monastery of Pedralbes (Catalonia) and the Cathedral of Altenberg (Germany): a comparative study, en "CVMA Newsletters" 47 (1999).

Deneux, H. A., A Thirteen Century Mould For Making Calme Lead, en "Journal of the British Society of Master Glass Painters" Vol. III, $n^{\circ} 2(1929)$, pp. 81-85.

Egan, G., Hanna, S. D. y Knight, B., Marks on Milled Window Leads, en "Post-Medieval Archacology", 20 (1986), pp. 303-309.

Fiaud. C., Le problème de l'altération des plombs anciens, en "Actes des Journées d'études du Centre International du Vitrail (Chartres)", Bourges, 28-29 October 1993, pp. 163-182.

Frenzel, G., Die Verbleiung Historischer Glasgemälde, en "C.V.M.A.“, 16. Intern. Kolloquium Bern, 1991, pp. 1-6.

Knight, B., Window Lead can be Interesting!, en "Conservation News", 29 (1986), pp. 31-32.

Knight, B., Researches on Medieval Window Lead, en "Journal of the British Society of Master Glass Painters" Vol. XVIII, $n^{\circ} 1$ (19831984), pp. 49-51.

Knowles, J. A., Ancient leads for windows and the methods of their manufacture, en "Journal of the British Society of Master Glass Painters"Vol. III, 3 (1930), pp. 133-140.

Knowles, J. A., Decay of glass, lead and iron of ancient stained glass windows, en "Journal of the British Society of Master Glass Painters"12,(1959), pp. 270-276.

Kratzel, W., Über Biege- und Zugversuche mit Proben der Verbleiung von Bildfenstern, en "Ö.Z.K.D.“, 21 (1967), pp. $205-207$.

Newton, R. G., Fact or fiction? Can cold glass flow under its own weight and what happens to stained glass windows?, en "Glass Technology“, vol. 37, nr. 4, Agosto 1996, p. 143.

Noehl, R., Korrosion von Fensterblei (Erstens frisches Blei, zweitens Nässe, drittens Säuren), en "Glaswelt" 4 (1991), pp. 25-26.

Pisa, A. da, Arte Delle Vetrate. End of the 14th century. Ed. Editalia, 1977. Introduction by Salvatore Pezzella.

Rambush, V. B., The lead cames of stained glass windows: purpose, problems and preservation procedures, en "Technology \& Conservation“, 8, N³ (1983), pp. 46-49.

Schmeling, E.- L., Lochfraßerscheinungen an Blei, en "Korrosion", 13 (1960), pp. 76-83.

Sloan, J., The best alloy for lead came, en "Professional Stained Glass" 171 (1989).

Strobel, S., Glastechnik des Mittelalters. Ed. Gentner Verlag, Stuttgart, 1990, pp. 112-123.

Theophilus, On Divers Arts. Traducido por Hawthorne y Smith, Nueva York, Ed. Dover Publications Inc., 1979, Libro II, pp. 67-71. 